\title{
SOME INEQUALITIES RELATED TO THE HAUSDORFF-YOUNG THEOREM
}

\author{
W. T. SLEDD
}

ABSTRACT. The classical Hausdorff-Young inequalities for Fourier series are extended. These new results are then used to improve known results on multipliers of $H^{p}$ spaces.

1. Introduction. In [4], C. N. Kellogg proved the following extensions of the classical Hausdorff-Young theorems.

(K) Let $1 \leqq p \leqq 2$ and $f \in H^{p}$ with $f(0)=0$. Then

$$
\sum_{m=1}^{\infty}\left(\sum_{n=2^{m^{-1}}}^{2^{m}-1}|\hat{f}(n)|^{p^{\prime}}\right)^{2 / p^{\prime}} \leqq A_{\nu}\|f\|_{p}^{2}
$$

where $\hat{f}(n)$ is the $n$th Fourier coefficient of $f$.

If

$$
S=\sum_{m=1}^{\infty}\left(\sum_{n=2^{m-1}}^{2^{m}-1}\left|a_{n}\right|^{p}\right)^{2 / p}<\infty
$$

then there is an $f \in H^{p^{\prime}}$ so that $\hat{f}(n)=a_{n}$ and $\|f\|_{p^{\prime}}^{2} \leqq A_{p} S$.

Kellogg used the results in $(\mathrm{K})$ to extend known results on multipliers of $H^{p}$ spaces.

It is the purpose of this note to extend Kellogg's results even further. In order to do this it will first be necessary to recall some known results. This will be done in the second section. The third section contains the extensions of Kellogg's results and the fourth section deals with some theorems on multipliers.

Regarding notation $r, p, q$ will denote real numbers satisfying $1<r$, $1<p \leqq 2 \leqq q$. Conjugate indices will be denoted by primes. All functions will be analytic and $\hat{f}(0)=0$. Constants depending on various parameters $p, q$ will be denoted by $A_{p q}$ and the usual abuse of notation will allow the same symbol to represent different constants.

The results of $\S \S 3$ and 4 , while stated for functions in $H^{r}$, may be extended to functions in $L^{r}$ when $r>1$ by using a well-known theorem of M. Riesz.

Received by the editors March 20, 1972 and, in revised form, May 11, 1973.

AMS (MOS) subject classifications (1970). Primary 42A18.

Key words and phrases. Multipliers of $H^{p}$ spaces, Littlewood-Paley theorems, Hausdorff-Young inequalities.

(c) American Mathematical Society 1974 
2. Known results. The first two results are due to Littlewood and Paley, and may be found in [5] or in [6, V.II, Chapters XIV, XV].

(LP I) Let $f \in H^{r}$ and $g(\theta)=\left(\int_{0}^{1}(1-\rho)\left|f^{\prime}\left(\rho e^{i \theta}\right)\right|^{2} d \rho\right)^{1 / 2}$. Then

$$
\|g\|_{r} \leqq A_{r}\|f\|_{r} \leqq A_{r}\|g\|_{r} .
$$

Then

(LP II) Let $f \in H^{r}$ and $\Delta_{m}=\Delta_{m}(f)=\sum_{n=2^{m-1}}^{2^{m}-1} \hat{f}(n) e^{i n \theta}, m=1,2, \cdots$.

$$
\left\|\left(\sum_{m=1}^{\infty}\left|\Delta_{m}\right|^{2}\right)^{1 / 2}\right\|_{r} \leqq A_{r}\|f\|_{r} \leqq A_{r}\left\|\left(\sum_{m=1}^{\infty}\left|\Delta_{m}\right|^{2}\right)^{1 / 2}\right\|_{r} .
$$

Various forms of Minkowski's inequality may be found in [2, pp. 148150]. These will be collectively referred to as (M).

Applying (M) to (LP I) gives two inequalities, which also may be found in [1].

$$
\begin{aligned}
& \int_{0}^{1}(1-\rho)\left(\int_{0}^{2 \pi}\left|f^{\prime}\left(\rho e^{i \theta}\right)\right|^{p} d \theta\right)^{2 / p} d \rho \leqq A_{\nu}\|f\|_{p}^{2}, \\
& \|f\|_{a}^{2} \leqq A_{a} \int_{0}^{1}(1-\rho)\left(\int_{0}^{2 \pi}\left|f^{\prime}\left(\rho e^{i \theta}\right)\right|^{a} d \theta\right)^{2 / a} d \rho .
\end{aligned}
$$

The next result is a corollary to the projection theorem of $M$. Riesz [6, V.I, p. 253].

(R) If $f$ is in $L^{r}$ then $\left\|\sum_{j}^{k} \hat{f}(n) e^{i n \theta}\right\|_{r} \leqq A_{r}\|f\|_{r}$ for any integers $k, j$.

Bernstein's theorem in the following form may be found in [6, V.II, p. 11].

(B) If $S$ is a trigonometric polynomial of degree $n$ then $\left\|S^{\prime}\right\|_{r} \leqq n\|S\|_{r}$.

\section{Some inequalities.}

(3.1) THEOREM. Same notation as in (LP II). Then

$$
\sum_{m=1}^{\infty}\left\|\Delta_{m}\right\|_{p}^{2} \leqq A_{p}\|f\|_{p}^{2} \text { and }\|f\|_{a}^{2} \leqq A_{a} \sum_{m=1}^{\infty}\left\|\Delta_{m}\right\|_{a}^{2}
$$

Proof. Since $1<p \leqq 2$, let $F(\theta, m)=\Delta_{m}$ and apply (M) and (LP II) to obtain the first inequality. A similar argument gives the second inequality.

An application of the classical Hausdorff-Young theorem [6, V.II, p. 101] shows that $(K)$ is contained in (3.1).

Next, it is shown that (3.1) may be sharpened.

(3.2) THEOREM. Let $M_{p}(\varphi, \rho)=\left(\int_{0}^{2 \pi}\left|\varphi\left(\rho e^{i \theta}\right)\right|^{p} d \theta\right)^{1 / p}$. Then

$$
\sum_{m=1}^{\infty}\left\|\Delta_{m}\right\|_{p}^{2} \leqq A_{p} \int_{0}^{1}(1-\rho) M_{p}^{2}\left(f^{\prime}, \rho\right) d \rho
$$


and

$$
\int_{0}^{1}(1-\rho) M_{q}^{2}\left(f^{\prime}, \rho\right) d \rho \leqq A_{a} \sum_{m=1}^{\infty}\left\|\Delta_{m}\right\|_{q}^{2}
$$

Proof. Note that (HL) shows that (3.2) is stronger than (3.1).

This proof illustrates a principle stated in [6, V.II, p. 223]. The principle says that $\Delta_{n}$ and $f\left(\rho_{n+1} e^{i \theta}\right)-f\left(\rho_{n} e^{i \theta}\right)$ behave alike if $\rho_{n}=1-2^{-n}$. Thus one expects that

$$
\left|\Delta_{n}\right| \leqq A\left|\int_{\rho_{n}}^{\rho_{n+1}} \frac{\partial f}{\partial \rho}\left(\rho e^{i \theta}\right) d \rho\right| \leqq A\left(\int_{\rho_{n}}^{\rho_{n+1}}\left|f^{\prime}\left(\rho e^{i \theta}\right)\right|^{p} d \theta\right)^{1 / p}\left(\rho_{n+1}-\rho_{n}\right)^{1 / p^{\prime}}
$$

so that

$$
\left\|\Delta_{n}\right\|_{D}^{2} \leqq A\left(\rho_{n+1}-\rho_{n}\right) \int_{\rho_{n}}^{\rho_{n+1}} M_{p}^{2}\left(f^{\prime}, \rho\right) d \rho \leqq A \int_{\rho_{n}}^{\rho_{n+1}}(1-\rho) M_{p}^{2}\left(f^{\prime}, \rho\right) d \rho,
$$

and so the first part of (3.2) would follow.

That it does is not nearly so difficult so show as the Littlewood-Paley theorems, but uses only the first few stages of that proof as found in $[6$, V.II, Chapter XV]. Since the steps are all included there many of the computations will be omitted.

Let $t_{m}(\rho, \theta)=\sum_{n=1}^{m} \hat{f}(n) \rho^{n} e^{i n \theta}, \quad t_{m}(\theta)=t_{m}(1, \theta)$. Then by Jensen's inequality

$$
\left|t_{m}^{\prime}(\theta)\right|^{p} \leqq \frac{A_{p}}{\rho^{m(p-1)}}\left[\frac{\left|t_{m}^{\prime}(\rho, \theta)\right|^{p}}{\rho^{m}}+(1-\rho) \sum_{n=1}^{m-1} \frac{\left|t_{n}^{\prime}(\rho, \theta)\right|^{p}}{\rho^{n+1}}\right] .
$$

By (R), $\int_{0}^{2 \pi}\left|t_{m}^{\prime}(\rho, \theta)\right|^{p} d \theta \leqq A_{p} \int_{0}^{2 \pi}\left|f^{\prime}\left(\rho e^{i \theta}\right)\right|^{p} d \theta$ and since the right-hand side increases with $\rho$

$$
\int_{0}^{2 \pi}\left|f^{\prime}\left(\rho e^{i \theta}\right)\right|^{p} d \theta \leqq \int_{0}^{2 \pi} \frac{1}{|I|} \int_{I}\left|f^{\prime}\left(t e^{i \theta}\right)\right|^{p} d t d \theta
$$

if $I$ is an interval contained in $(\rho, 1)$. Combining these results

$$
\int_{0}^{2 \pi}\left|t_{m}^{\prime}(\theta)\right|^{\nu} d \theta \leqq \frac{A_{p}}{\rho^{m \nu}} \int_{0}^{2 \pi} \frac{1}{|I|} \int_{I}\left|f^{\prime}\left(t e^{i \theta}\right)\right|^{\nu} d t d \theta .
$$

Now let $\rho=\rho_{m}=1-(m+1)^{-1}$, and $I=I_{m}=\left(\rho_{m}, \rho_{m+1}\right)$. Then

$$
\left\|t_{m}^{\prime}\right\|_{p}^{2} \leqq A_{p} \frac{1}{\left|I_{m}\right|} \int_{I_{m}} M_{p}^{2}\left(f^{\prime}, \rho\right) d \rho \leqq A_{p} m^{3} \int_{I_{m}}(1-\rho) M_{p}^{2}\left(f^{\prime}, \rho\right) d \rho .
$$

Next, let $\rho=\rho_{m}^{*}=1-2^{-m}, I=I_{m}^{*}=\left(\rho_{m}^{*}, \rho_{m+1}^{*}\right)$. Then

$$
\left\|t_{2}^{\prime}{ }^{m}-1\right\|_{p}^{2} \leqq A_{p} 2^{2 m} \int_{I_{m}^{*}}(1-\rho) M_{p}^{2}\left(f^{\prime}, \rho\right) d \rho
$$


By combining Abel partial summation with some standard inequalities (see [6, V.II, p. 228]),

$$
\left\|\Delta_{m}\right\|_{p}^{2} \leqq A_{p}\left[\frac{\left\|t_{2}^{\prime m}-1\right\|_{p}^{2}}{2^{2 m}}+\sum_{2^{m-1}}^{2^{m}-2} \frac{\left\|t_{n}^{\prime}\right\|_{p}^{2}}{n^{3}}+\frac{\left\|t_{2}^{\prime m-1}-1\right\|_{p}^{2}}{2^{2(m-1)}}\right]
$$

and this together with the preceding results completes the proof of the first part of (3.2).

Now for the second part of (3.2). Most of the argument is very similar to Lemma (3.1) of [6, V.II, p. 229] and so again many of the computations are omitted. Since $\left|f^{\prime}\left(\rho e^{i \theta}\right)\right|=\left|(1-\rho) \sum_{n=1}^{\infty} t_{n}^{\prime} \rho^{n-1}\right|$, where $t_{n}=t_{n}(\theta)$, then $\int_{0}^{1}(1-\rho) M_{a}^{2}\left(f^{\prime}, \rho\right) d \rho$ is dominated by

$$
\begin{gathered}
\sum_{m=0}^{\infty} \int_{I_{m}}(1-\rho)^{3}\left[\left(\int_{0}^{2 \pi}\left|\sum_{n=1}^{m+1} t_{n}^{\prime} \rho^{n-1}\right|^{Q} d \theta\right)^{2 / Q}+\left(\int_{0}^{2 \pi}\left|\sum_{n=m+2}^{\infty} t_{n}^{\prime} \rho^{n-1}\right|^{q} d \theta\right)^{2 / \alpha}\right] d \rho \\
=P+Q,
\end{gathered}
$$

where again $\rho_{m}=1-(m+1)^{-1}$ and $I_{m}=\left(\rho_{m}, \rho_{m+1}\right)$. But

and

$$
\begin{aligned}
P & \leqq A \sum_{m=0}^{\infty} \frac{1}{(m+1)^{3}} \int_{I_{m}}\left(\sum_{n=1}^{m+1}\left\|t_{n}^{\prime}\right\|_{a} \rho^{n-1}\right)^{2} d \rho \\
& \leqq A \sum_{m=0}^{\infty} \frac{1}{(m+1)^{5}} \sum_{n=1}^{m+1}\left\|t_{n}^{\prime}\right\|_{a}^{2}(m+1) \leqq A \sum_{n=1}^{\infty} \frac{\left\|t_{n}^{\prime}\right\|_{a}^{2}}{n^{3}}
\end{aligned}
$$

so that

$$
\begin{aligned}
Q & \leqq A \sum_{m=0}^{\infty} \frac{1}{(m+1)^{3}} \int_{I_{m}}\left(\sum_{n=m+2}^{\infty}\left\|t_{n}^{\prime}\right\|_{a} \rho^{n-1}\right)^{2} d \rho \\
& \leqq A \sum_{m=0}^{\infty} \frac{1}{(m+1)^{5}} \sum_{n=m+2}^{\infty} \frac{\left\|t_{n}^{\prime}\right\|_{a}^{2}}{n^{4}} \sum_{n=m+2}^{\infty} n^{4} \rho_{m}^{2(n-1)} \\
& \leqq A \sum_{m=0}^{\infty} \frac{1}{(m+1)^{5}} \frac{1}{\left(1-\rho_{m}\right)^{5}} \sum_{n=m+2}^{\infty} \frac{\left\|t_{n}^{\prime}\right\|_{a}^{2}}{n^{4}} \\
& \leqq A \sum_{n=1}^{\infty} \frac{\left\|t_{n}^{\prime}\right\|_{a}^{2}}{n^{3}}
\end{aligned}
$$

$$
\int_{0}^{1}(1-\rho) M_{q}^{2}\left(f^{\prime}, \rho\right) d \rho \leqq A_{a} \sum_{n=1}^{\infty} \frac{\left\|t_{n}^{\prime}\right\|_{q}^{2}}{n^{3}} .
$$

But applying (3.1) and (R), $\left\|t_{n}^{\prime}\right\|_{a}^{2} \leqq A_{a} \sum_{k=1}^{j}\left\|\Delta_{k}^{\prime}\right\|_{a}^{2}$ if $2^{j-1} \leqq n<2^{j}$, and by (B) $\left\|\Delta_{k}^{\prime}\right\|_{e}^{2} \leqq 2^{2 k}\left\|\Delta_{k}\right\|_{Q}^{2}$ so that

$$
\begin{aligned}
\sum_{n=1}^{\infty} \frac{\left\|t_{n}^{\prime}\right\|_{a}^{2}}{n^{3}} & \leqq A_{a} \sum_{j=1}^{\infty} \sum_{n=2^{j-1}}^{2^{j}-1} \frac{1}{n^{3}} \sum_{k=1}^{j}\left\|\Delta_{k}^{\prime}\right\|_{a}^{2} \\
& \leqq A_{q} \sum_{k=1}^{\infty}\left\|\Delta_{k}^{\prime}\right\|_{a}^{2} \sum_{j=k}^{\infty} \frac{1}{2^{2 j}} \leqq A_{a} \sum_{k=1}^{\infty} \frac{\left\|\Delta_{k}^{\prime}\right\|_{q}^{2}}{2^{2 k}} \leqq A_{a} \sum_{k=1}^{\infty}\left\|\Delta_{k}\right\|_{q}^{2}
\end{aligned}
$$


4. Applications to the multipliers. If $\lambda=(\lambda(n))$ is a sequence of complex numbers with the property that whenever $f \in H^{r}$ there is a $g \in H^{s}$ so that $\hat{g}(n)=\lambda(n) \hat{f}(n)$ then $\lambda$ is called a multiplier of $H^{r}$ into $H^{s}$, or $\lambda \in\left(H^{r}, H^{s}\right)$.

(4.1) Theorem. Let $I_{n m}$ denote the mth dyadic-subinterval of $I_{n}=$ $\left[2^{n-1}, 2^{n}-1\right)$ and

$$
\sum_{I_{n m}} a_{n} e^{i n \theta}=\Delta_{n m}
$$

If $\sum_{I_{n m}}|\lambda(k)|^{s} \leqq A$ where $1 / s=1 / p-1 / q$ then $\lambda \in\left(H^{p}, H^{q}\right)$.

Proof. The proof is a direct application of (3.1) and the classical Hausdorff-Young theorem.

The hypotheses imply (see [4]) that

$$
\left(\sum_{I_{n m}}|\lambda(k) \hat{f}(k)|^{q^{\prime}}\right)^{2 / q^{\prime}} \leqq\left(\sum_{I_{n m}}|\lambda(k)|^{s}\right)^{2 / s}\left(\sum_{I_{n m}}|\hat{f}(k)|^{p^{\prime}}\right)^{2 / p^{\prime}} .
$$

So if $\hat{g}(n)=\lambda(n) \hat{f}(n)$ then applying (3.1) twice,

$$
\|g\|_{a}^{2} \leqq A_{a} \sum_{n=1}^{\infty}\left\|\Delta_{n}(g)\right\|_{a}^{2} \leqq A_{a} \sum_{n=1}^{\infty} \sum_{m}\left\|\Delta_{n m}(g)\right\|_{a}^{2} .
$$

But $\Delta_{n m}(g)=\sum I_{n m} \hat{g}(k) e^{i k \theta}$. So by the Hausdorff-Young theorem

$$
\begin{aligned}
\|g\|_{a}^{2} & \leqq A_{a} \sum_{n=1}^{\infty} \sum_{m}\left(\sum_{I_{n m}}|\hat{g}(k)|^{q^{\prime}}\right)^{2 / q^{\prime}} \\
& \leqq A_{a} \sum_{n} \sum_{m}\left(\sum_{I_{n m}}|\hat{f}(k)|^{p^{\prime}}\right)^{2 / p^{\prime}} \\
& \leqq A_{p a} \sum_{n} \sum_{m}\left\|\Delta_{n m}(f)\right\|_{p}^{2} \leqq A_{p a} \sum_{n}\left\|\Delta_{n}(f)\right\|_{p}^{2} \leqq A_{p q}\|f\|_{p}^{2},
\end{aligned}
$$

where (3.1) has again been applied twice. This completes the proof of (4.1).

Similarly if $I_{n m k}$ is the $k$ th dyadic subinterval of $I_{n m}$ then whenever $\sum_{I_{n m k}}|\lambda(j)|^{s} \leqq A<\infty$ it follows that $\lambda \in\left(H^{p}, H^{q}\right)$. And this kind of result may be improved over and over.

At the same time it gives a sequence of improvements over the Hausdorff-Young theorems. In the case above, for example, one gets $\sum_{n, m}\left(\sum_{I_{n m}}|\hat{f}(k)|^{p^{\prime}}\right)^{2 / p^{\prime}} \leqq A_{p}\|f\|_{p}^{2}$, etc.

None of this seems to go over to $H^{1}$, however. For instance $\lambda \in\left(H^{1}, H^{2}\right)$ if and only if $\sum_{2^{m-1}}^{2^{m}-1}|\lambda(n)|^{2} \leqq A$ (see [3]), so this case admits no weakening of the type described above.

All the multiplier theorems of the type thus far described are actually multiplier theorems for sequence spaces that are related to $H^{r}$ (or $L^{r}$ ) 
but only approximate them. Thus (4.1) or anything like it seems unlikely to be definitive. But the results in [3], [4] and $\$ 3$ and $\$ 4$ of the present paper do show that any theorems on $H^{r}$-multipliers may be localized. For instance it is only necessary (with the terminology of (4.1)) to show that

$$
\left\|\Delta_{n m}(g)\right\|_{Q} \leqq\left\|\Delta_{n m}(f)\right\|_{p}
$$

in order to have $\lambda$ in $\left(H^{p}, H^{q}\right)$.

Thus, for instance, this observation and a direct application of Theorem 1 of [1] give the following extension of that theorem.

(4.2) THEOREM. If $1 / s=1 / q-1 / p+1$ and $h$ is an integrable function that satisfies

$$
M_{s}\left(\Delta_{n m}\left(h^{\prime}\right), \rho\right) \leqq A /(1-p)
$$

then $(\hat{h}(n)) \in\left(H^{p}, H^{q}\right)$.

Proof. For then if $f \in H^{p}$ and $\hat{g}(k)=\hat{h}(k) \hat{f}(k)$ then $\left\|\Delta_{n m}(g)\right\|_{a} \leqq$ $A_{p q}\left\|\Delta_{n m}(f)\right\|_{p}$ by the theorem cited above and so by an argument similar to (4.1) $\|g\|_{q} \leqq A_{p q}\|f\|_{p}$.

\section{REFERENCES}

1. G. H. Hardy and J. E. Littlewood, Notes on the theory of series. XX: Generalizations of a theorem of Paley, Quart. J. Math. Oxford Ser. 8 (1937), 161-171.

2. G. H. Hardy, J. E. Littlewood and G. Polya, Inequalities, Cambridge Univ. Press, New York, 1934.

3. J. H. Hedlund, Multipliers of $H^{p}$ spaces, J. Math. Mech. 18 (1968/69), 10671074. MR 39 \#4404.

4. C. N. Kellogg, An extension of the Hausdorff-Young theorem, Michigan Math. J. 18 (1971), pp. 121-127. MR 43 \#6714.

5. J. E. Littlewood and R. E. A. C. Paley, Theorems on Fourier series and power series. II, Proc. London Math. Soc. 42 (1936), 52-89.

6. A. Zygmund, Trigonometric series, 2nd rev. ed., Vol. I, II, Cambridge Univ. Press, New York, 1959. MR 21 \#6498.

Department of Mathematics, Michigan State University, East Lansing, MiCHIGAN 48823 